\title{
Tratamento precoce para COVID-19 baseado em evidência científica
}

\section{Early treatment of COVID-19 based on scientific evidence \\ Tratamiento precoz para COVID-19 basado en evidencia científica}

\author{
Sabas Carlos Vieira (iD \\ Universidade Federal do Piauí - Teresina (PI) - Brasil \\ Oncocenter - Teresina (PI) - Brasil \\ Danilo Rafael da Silva Fontinele (iD) \\ Universidade Estadual do Piauí - Teresina (PI) - Brasil \\ Marina Bucar Barjud (iD \\ Hospital Universitário HM Puerta del Sur - Madrid - Espanha
}

Justino Moreira de Carvalho Junior

Hospital Regional Tibério Nunes - Floriano (PI) - Brasil

Lina Madeira Campos Melo iD

Hospital de Terapia Intensiva - Teresina (PI) - Brasil

José Wilson Fonseca Filho iD

Hospital Otorrinos - Teresina (PI) - Brasil

\section{Gerson Luís Medina Prado iD}

Universidade Federal do Piauí - Teresina (PI) - Brasil

Hospital de Urgência de Teresina - Teresina (PI) - Brasil

\author{
Alexandre Adad Alencar iD \\ Universidade Federal do Piauí - Teresina (PI) - Brasil \\ Hospital Getúlio Vargas - Teresina (PI) - Brasil
}

\section{RESUMO}

Objetivo: Apresentar uma proposta de tratamento precoce para pacientes com COVID-19 em Unidade Básica de Saúde (UBS). Métodos: Trata-se de uma proposta de protocolo de tratamento para COVID-19 com hidroxicloroquina ou cloroquina com base na literatura científica disponível no momento (13 de junho de 2020). O levantamento dos estudos ocorreu na base PubMed, por meio dos descritores COVID-19, hydroxychloroquine e chloroquine. Foram selecionados cinco artigos, dos 645 artigos encontrados, por abordarem o uso de hidroxicloroquina ou cloroquina no tratamento da fase precoce da COVID-19. Resultados: A proposta de protocolo para tratamento precoce para pacientes com suspeita de COVID-19 envolve orientações sobre os principais sintomas, orientações gerais à população, avaliação médica, considerações sobre exames, terapia recomendada, orientações pósprescrição e critérios para internação. Conclusão: Baseados em estudos, o tratamento precoce ambulatorial da COVID-19 com hidroxicloroquina ou cloroquina parece diminuir o risco de internação e, assim, a necessidade de leitos de terapia intensiva. A decisão de adoção do tratamento a ser proposto é do paciente, e o médico só deve adotar após o paciente assinar o consentimento livre e esclarecido, por tratar-se de prescrição off label. Estudos randomizados são necessários para confirmar essa hipótese.

Descritores: Protocolos; Terapêutica; Infecções por Coronavirus.

\section{ABSTRACT}

Objective: To present a proposal for early treatment of patients with COVID-19 in a Primary Health Care (PHC) center. Methods: This is a proposed treatment protocol for COVID-19 using hydroxychloroquine or chloroquine based on the currently (June 13, 2020) available scientific literature. PubMed database was searched for studies using the descriptors COVID-19, hydroxychloroquine, 
and chloroquine. Five studies were selected from the 645 studies found as they addressed the use of hydroxychloroquine or chloroquine in the treatment of COVID-19 in early stages. Results: The proposed early treatment protocol for patients with suspected COVID-19 includes guidance on the main symptoms, general guidelines for the population, medical evaluation, examination considerations, recommended therapy, post-prescription guidelines and criteria for hospitalization. Conclusion: According to the studies, early outpatient treatment of COVID-19 with hydroxychloroquine or chloroquine seems to decrease the risk of hospitalization and the need for intensive care beds. The decision to adopt the proposed treatment is made by the patient, and the physician should only conduct the treatment after obtaining written informed consent from the patient, as it is an off-label prescription. Randomized studies are needed to confirm this hypothesis.

Descriptors: Protocols; Therapeutics; Coronavirus Infections.

\section{RESUMEN}

Objetivo: Presentar una propuesta de tratamiento precoz para pacientes con la COVID-19 en Unidad Básica de Salud (UBS). Métodos: Se trata de una propuesta de protocolo de tratamiento para la COVID-19 con hidrocloroquina o cloroquina basado en la literatura científica disponible en el momento (13 de junio de 2020). La recogida de los estudios se dio en la base de datos PubMed a través de los descriptores COVID-19, hydroxychloroquine y chloroquine. De los 645 artículos encontrados se ha elegido cinco de ellos sobre el uso de la hidrocloroquina o cloroquina para el tratamiento de la fase precoz de la COVID-19. Resultados: En la propuesta del protocolo para el tratamiento precoz de pacientes con sospecha de la COVID-19 hay orientaciones de los síntomas principales, las orientaciones generales para la población, la evaluación médica, las consideraciones de las pruebas, la terapia recomendada, las orientaciones post prescripición y los criterios para el ingreso hospitalario. Conclusión: Basados en estudios, el tratamiento precoz de ambulatorio de la COVID-19 con hidrocloroquina o cloroquina parece disminuir el riesgo de ingreso hospitalario y la necesidad de camas de la unidad de cuidados intensivos. La decisión para la adhesión al tratamiento propuesto es del paciente y el medico solo debe iniciarlo tras la firma del consentimiento libre esclarecido de parte del paciente una vez que se trata de prescripción off label. Son necesarios estudios randomizados para confirmar esa hipótesis.

Descriptores: Protocolos; Terapéutica; Infecciones por Coronavirus.

\section{INTRODUÇÃO}

A crescente pandemia de COVID-19 está gerando uma crise global que já se tornou um desastre de saúde, econômico e humanitário. O SARS-CoV-2 é um betacoronavírus descrito pela primeira vez após o diagnóstico de pneumonia de causa desconhecida na província de Wuhan, na China, em novembro de $2019^{(1)}$.

O sequenciamento do vírus foi divulgado em 10 de janeiro de 2020, cujo genoma agora está disponível no GenBank ${ }^{(2)}$. Trata-se de um vírus de RNA simples que apresenta espículas em sua superfície, vista à microscopia eletrônica, lembrando uma coroa. O SARS-CoV-2 infecta, predominantemente, as vias áreas inferiores, ligando-se aos receptores das células epiteliais alveolares, induzindo intensa resposta de citocinas inflamatórias. A tempestade de citocinas é o mecanismo postulado para lesão de órgãos ${ }^{(3,4)}$.

A Organização Mundial de Saúde (OMS) declarou, em 30 de janeiro de 2020, que a COVID-19 era uma emergência global, e, em 11 de março de 2020, declarou que a doença já se tratava de uma nova pandemia, com muitos países já atingidos ${ }^{(5)}$.

No mundo, até o dia 12 de junho de 2020, às $21 \mathrm{~h}$, haviam 7.553 .182 casos confirmados, com 423.349 mortes e 216 países, áreas ou territórios atingidos pela COVID-19(6). No Brasil, até o dia 12 de junho de 2020, às 18h30, haviam 828.810 casos confirmados, 25.982 casos novos e 41.828 óbitos, sendo 909 novos casos de óbitos. Felizmente, também até o dia 12 de junho de 2020, no mesmo horário, já haviam no país 365.063 casos recuperados e outros 421.919 ainda seguiam em acompanhamento ${ }^{(7)}$.

A COVID-19 é, predominantemente, uma doença respiratória, com gravidade variando de leve a fatal, e a transmissão ocorre principalmente por disseminação de gotículas respiratórias ${ }^{(8)}$. Além das gotículas no ar, o vírus SARS-CoV-2 pode sobreviver por até quatro dias quando essas gotículas respiratórias se depositam nas superfícies, com base em experimentos in vitro ${ }^{(9)}$.

Essas características fazem com que o vírus tenha alta transmissividade. Em modelos matemáticos baseados nos brotes epidêmicos de Singapura e Tiajin (China), estimou-se que a transmissão a partir de casos pré-sintomáticos e sintomáticos seja de 45\% (IC 95\% 32-67) e 62\% (IC 95\% 50-76), respectivamente ${ }^{(10)}$.

Estudos chineses estimam que a taxa de mortalidade da COVID-19 seja de 0,25-3\%(11), entretanto, dada a sua alta taxa de transmissão, é considerada pela avaliação de riscos da OMS como um "risco muito alto em nível 
global”(5). No Brasil, a taxa de letalidade da COVID-19 em dados publicados no dia 12 de junho de 2020, às 20h15, pelo Ministério da Saúde, foi $5,0 \%{ }^{(7)}$.

A COVID-19 evolui em fases clínicas bem definidas: Fase I, Fase II e Fase III. A Fase I (infecção leve/ infeç̧ão precoce) ocorre no momento da inoculação e estabelecimento precoce da doença. Para a maioria dos pacientes infectados, isso envolve um período de incubação associado a sintomas leves e, geralmente, inespecíficos, como mal-estar, febre e tosse seca ${ }^{(12)}$.

A Fase II (moderada, com envolvimento pulmonar sem hipóxia - IIA e com envolvimento pulmonar e hipóxia - IIB) é o estágio em que a doença pulmonar é estabelecida e a multiplicação viral e a inflamação localizada no pulmão são a norma. Nessa fase, os pacientes desenvolvem pneumonia viral, com tosse, febre e hipóxia na fase IIB (definida como $\mathrm{PaO} 2$ / FiO2 $<300 \mathrm{mmHg}$ ). A imagem com radiografia de tórax ou tomografia computadorizada revela infiltrados bilaterais ou opacidades em vidro fosco. Os exames de sangue revelam aumento da linfopenia e de transaminases. Marcadores de inflamação sistêmica podem estar elevados, mas não notavelmente. É nessa fase que a maioria dos pacientes com COVID-19 precisa ser hospitalizados para observação e tratamento ${ }^{(12)}$.

Já na Fase III (grave) ocorre uma hiperinflamação sistêmica. Uma minoria de pacientes com COVID-19 fará a transição direta para essa fase da doença, que se manifesta como uma síndrome de hiperinflamação sistêmica extrapulmonar. Nesse estágio, os marcadores de inflamação sistêmica se elevam de forma significativa. Uma forma semelhante à linfohistiocitose hemofagocítica $(\mathrm{sHLH})$ pode ocorrer em pacientes nesse estágio avançado da doença, além de choque, vasoplegia, insuficiência respiratória, miocardite e colapso cardiopulmonar. A terapia na Fase III depende do uso de agentes imunomoduladores para reduzir a inflamação sistêmica antes que evolua para disfunção de múltiplos órgãos e sistemas, chegando ao óbito(12).

A fase infecciosa ou de replicação viral da doença geralmente dura, mais ou menos, sete dias. A partir do $7^{\circ}-10^{\circ}$ dia, o paciente entra na fase inflamatória ou de inflamação pulmonar ${ }^{(13)}$. Na primeira fase de inflamação pulmonar (IIA), não existe hipóxia, ou seja, o paciente não sente dispneia, mas já existe inflamação pulmonar, isto é, o processo de tempestade citoquímica já está se iniciando(12).

Um agravante que, na prática clínica, se observa são pacientes com sensação dispneica desproporcional em relação à imagem radiológica. Além disso, ainda não foi identificado nenhum marcador prognóstico fidedigno que detecte os pacientes que evoluirão para a fase de inflamação pulmonar. De acordo com uma revisão sistemática, os preditores mais relatados de prognóstico grave em pacientes com COVID-19 são: idade, sexo, características derivadas de tomografias computadorizadas, presença de proteína C-reativa, desidrogenase láctica e contagem de linfócitos, mas os autores ponderam que modelos de predição mais acurados são necessários para evitar excesso ou subtratamentos que possam causar riscos desnecessários ou comprometer o prognóstico. Esses fatos ratificam a necessidade de uma boa orientação aos pacientes para acessar o serviço de saúde antes da dispneia e iniciar precocemente o tratamento ${ }^{(14)}$.

Por um lado, embora não exista nenhum tratamento especifico para o SARS-CoV-2 até o momento, várias alternativas têm sido propostas: antivirais, hidroxicloroquina, cloroquina, corticosteroide, tocizilumabe, ivermectina, imunoglobulina e plasmaférese, entre outras ${ }^{(15-18)}$.

Por outro lado, para o tratamento nas fases avançadas da doença são necessários recursos financeiros e físicos insustentáveis, em um contexto de pandemia, para qualquer país do mundo. Dessa forma, as estratégias mais eficientes seriam a prevenção e o tratamento precoce da doença. Os dados iniciais com o tratamento precoce são promissores em diminuir a gravidade da doença e a necessidade de leitos de terapia intensiva ${ }^{(19)}$. Além disso, estudos randomizados e observacionais importantes ${ }^{(20-25)}$, que utilizaram cloroquina ou hidroxicloroquina na Fase II e III da COVID-19, não demonstraram diminuição da mortalidade e, em um desses estudos ${ }^{(21)}$, que utilizou $12 \mathrm{~g}$ de cloroquina, houve um aumento da mortalidade.

No presente trabalho, será avaliado o papel da hidroxicloroquina e da cloroquina no tratamento da Fase I da COVID-19 com o objetivo de apresentar uma proposta de tratamento precoce em Unidades Básicas de Saúde (UBS).

\section{MÉTODOS}

Trata-se de uma proposta de protocolo para tratamento precoce de pacientes com COVID-19, realizada por meio de um estudo bibliométrico exploratório da literatura científica disponível no momento (13 de junho de 2020), utilizando a hidroxicloroquina ou cloroquina, associada ou não a um macrolídeo.

O levantamento dos estudos ocorreu na PubMed, por meio dos descritores controlados e não controlados: COVID-19, hydroxychloroquine e chloroquine, associados pelos operadores booleanos OR e AND. Foram encontrados 
645 artigos: cinco ensaios clínicos, sendo três estudos randomizados; 34 estudos de caso, entre outros. Foram selecionados somente os cinco artigos que abordavam o uso de hidroxicloroquina ou cloroquina, associado ou não a macrolídeos, no tratamento da fase precoce da COVID-19. Além disso, foi incluído um artigo da plataforma medRxiv (trabalhos não certificados por revisão por pares) por se tratar de um estudo randomizado.

A seguir, são apresentados os estudos selecionados que guiaram a elaboração da proposta de tratamento precoce para pacientes com COVID-19.

Um dos primeiros estudos publicados foi na forma de carta, que reportou "mais" de 100 pacientes tratados com cloroquina $500 \mathrm{mg}$, de 12/12 horas, por via oral para pneumonia por SARS-Cov-2. Os pacientes que receberam cloroquina apresentaram melhora significativa dos sintomas e da imagem radiológica em comparação aos controles que não utilizaram. Os autores relatam, ainda, que o protocolo foi incorporado ao Guideline Chinês de COVID-19 após uma reunião em 15 de fevereiro de 2020 com autoridades governamentais e regulatórias ${ }^{(26)}$. No entanto, nesse estudo, os autores não apresentaram as taxas de complicações e a taxa de mortalidade, bem como os detalhes clínicos dos pacientes de cada grupo.

Outro estudo, este randomizado, porém publicado na forma de preprint, incluiu 62 pacientes e avaliou a administração de hidroxicloroquina $400 \mathrm{mg} / \mathrm{dia}$, por cinco dias, ou placebo em pacientes com COVID-19. Os autores avaliaram os achados radiológicos na admissão e, cinco dias depois, todos os pacientes estavam internados em enfermaria. Além da hidroxicloroquina, grupo de intervenção, todos os pacientes receberam oxigênio, agentes antivirais, antibiótico e imunoglobulina, com ou sem corticoide. A recuperação da tosse e da febre foi mais rápida no grupo de intervenção com hidroxicloroquina. A melhora radiológica da pneumonia no grupo de intervenção foi de $80,6 \%$ e, no de controle, $54,5 \%$. Os quatro pacientes que evoluíram com piora foram do grupo controle. Apenas dois efeitos adversos foram relatados: um caso de cefaleia e um rash cutâneo. Não foi reportada a taxa de mortalidade e os dias de internação nesse estudo(18).

Um pequeno estudo observacional(17) francês avaliou o uso da hidroxicloroquina $600 \mathrm{mg} / \mathrm{dia}$, associada ou não a azitromicina, em 20 pacientes. Avaliaram o clareamento viral através de RT-PCR (Reverse Transcription Polymerase Chain Reaction) obtido de coleta de swab nasofaríngeo. Houve eliminação do vírus em $100 \%$ dos casos de pacientes que utilizaram hidroxicloroquina e azitromicina até o sexto dia, já os que usaram somente hidroxicloroquina, $57,1 \%$, e nos de controle a eliminação completa do vírus ocorreu em $12,5 \%$ no sexto dia. Não foram relatados outros desfechos importantes, como mortalidade e dias de internação.

Em outro estudo observacional não controlado( ${ }^{(27)}$, com uma coorte de 80 pacientes com infecção relativamente leve e tratados com uma combinação de hidroxicloroquina e azitromicina, por um período de pelo menos três dias, todos os pacientes melhoraram clinicamente, exceto um paciente de 86 anos que foi a óbito e um paciente de 74 anos que ainda estava em terapia intensiva. Houve uma queda rápida da carga viral nasofaríngea, com $83 \%$ de negativos no $7^{\circ}$ dia e $93 \%$ no $8^{\circ}$ dia. As culturas do vírus das amostras respiratórias dos pacientes foram negativas em $97,5 \%$ dos pacientes no $5^{\circ}$ dia. Os pacientes puderam receber alta rapidamente da unidade de doença infecciosa, com um tempo médio de permanência de cinco dias.

O principal estudo publicado até o momento, que avaliou o tratamento precoce na Fase I da COVID-19, foi o estudo realizado em Marseille, na França ${ }^{(19)}$. Os autores avaliaram 1.061 positivos para SARS-CoV-2 por RTPCR colhido de swab nasal, tratados por pelo menos três dias com hidroxicloroquina ( $200 \mathrm{mg}$ três vezes ao dia por dez dias) e azitromicina (500 mg no primeiro dia, seguido por $250 \mathrm{mg}$ por dia nos quatro dias subsequentes). O clareamento viral determinado por RT-PCT ocorreu em 973 pacientes em 10 dias (91,7\%). O resultado clínico adverso (admissão na Unidade de Terapia Intensiva - UTI, óbito ou hospitalização por 10 dias ou mais) foi observado em 46 pacientes $(4,3 \%)$ e 8 foram a óbito $(0,75 \%$, com idades de 74 a 95 anos). Todas as mortes resultaram de insuficiência respiratória e não de toxicidade cardíaca.

Cinco pacientes ainda estavam internados (entretanto com 98,7\% dos pacientes curados) no momento da submissão do estudo para publicação. O resultado clínico adverso foi associado à: idade avançada (OR 1,11), gravidade da doença na admissão (OR 10,05) e baixa concentração sérica de hidroxicloroquina. O mau resultado clínico foi associado, de forma independente, também ao uso de agentes betabloqueadores seletivos e bloqueadores dos receptores da angiotensina II $(p<0,05)$. Um total de $2,3 \%$ dos pacientes relatou eventos adversos leves (sintomas gastrointestinais ou cutâneos, dor de cabeça, insônia e visão turva transitória).

Assim, a administração da combinação hidroxicloroquina e azitromicina antes da ocorrência de complicações do COVID-19 é segura e associada a uma taxa de mortalidade muito baixa nos pacientes. Embora o estudo não seja randomizado e também não tenha realizado comparação com um grupo controle histórico, sem utilizar hidroxicloroquina, gera-se a hipótese de que o tratamento precoce poderia diminuir a internação na enfermaria e na UTI, e a taxa de óbito. Esses dados precisam ser confirmados em estudos randomizados controlados. 
Também baseados na experiência clínica, o grupo de colaboração multicêntrica do Departamento de Ciência e Tecnologia da província de Guangdong e da Comissão de Saúde da província de Guangdong descreve que tratar os pacientes diagnosticados com pneumonia por coronavírus com cloroquina melhora a taxa de sucesso do tratamento, diminui a permanência média e diminui a probabilidade de sequelas nos pacientes. A fim de orientar e regular o uso de cloroquina em pacientes com nova pneumonia por SARS-CoV-2, o grupo desenvolveu um documento de consenso, após extensa discussão, no qual recomenda comprimido de fosfato de cloroquina para pacientes diagnosticados como casos leves, moderados e graves de nova pneumonia por SARS-CoV-2 e sem contraindicações à cloroquina ${ }^{(28)}$.

Vale lembrar que não existem evidências cientificas nível 1A sobre a eficácia ou a contraindicação da hidroxicloroquina, como visto nos estudos apresentados. Nos últimos estudos que contraindicaram o uso da medicação, a droga não foi utilizada na Fase I da doença ${ }^{(20-25)}$. Assim, o uso da hidroxicloroquina na Fase I é uma opção.

Nesse contexto, apresenta-se uma proposta de protocolo cujo principal objetivo é contribuir com estratégias que possam evitar ou diminuir a probabilidade de um paciente com COVID-19, na fase infecciosa ou de replicação viral, evoluir para a fase inflamatória, em que as probabilidades de recuperação são menores no contexto atual e, assim, diminuir a mortalidade e a probabilidade de sequelas permanentes, como fibrose pulmonar, nesses pacientes, além de tentar contribuir para diminuir a demanda por leitos de enfermaria e UTI.

Outra consideração sobre o tratamento precoce é tentar diminuir a sintomatologia aguda naqueles pacientes que, estatisticamente, teriam doença autolimitada.

\section{RESULTADOS}

A seguir, apresenta-se a proposta de protocolo de tratamento precoce para pacientes com suspeita de COVID-19 na Fase I da doença (Tabela I).

Tabela I - Protocolo para tratamento precoce da COVID-19.

Principais sintomas clínicos (síndrome gripal)

Febre; tosse seca; cefaleia persistente; desconforto na garganta São sinais muito sugestivos de COVID19: febre persistente (90\% ("bolo ao engolir"); mialgia, dor torácica; astenia; náuseas, vômitos dos casos), associado à tosse seca e sintomas gastrointestinais. e diarreia; anosmia (até $20-25 \%$ dos pacientes apresentam perda Pacientes com anosmia, mais de $80 \%$ dos casos, têm PCR positivo do olfato); ageusia (perda do paladar) ${ }^{(3,8,13,29)}$. para SARS-CoV-2, portanto é um sintoma a ser valorizado ${ }^{(3,8,13)}$.

\section{Momento de procurar assistência médica}

Em caso de febre e dois ou mais desses sintomas que persistam sem melhora por mais de 3 a 4 dias, o paciente deve ser orientado a procurar assistência médica ${ }^{(19)}$.

Se a febre desaparecer, mas os outros sintomas não melhorarem, pode ser um dos sinais de que o paciente está entrando na fase de inflamação e requer atenção redobrada do médico para não perder a janela de oportunidade do tratamento precoce ${ }^{(19)}$.

\section{Avaliação médica no pronto atendimento}

Idealmente, realizar avaliação clínica, radiológica e laboratorial ${ }^{(8)}$.

Caso seja impossível a realização de todos os exames ideais, considerando a situação de pandemia e o risco de evolução desfavorável e rápida, recomenda-se esclarecimento ao paciente e aos familiares e solicitação de assinatura de consentimento informado para instituir tratamento precoce com hidroxicloroquina e azitromicina ${ }^{(29)}$.

As evidências científicas, no momento, são de pequenos estudos com metodologias inapropriadas. Estudos randomizados estão em andamento em vários países e, assim que publicados, as recomendações poderão ser atualizadas ${ }^{(29)}$.

Prescrição imediata de esquema terapêutico recomendado para o tratamento de COVID19(29).

\section{Considerações sobre exames}

Pacientes portadores de fatores de risco: idade >60anos, HAS (hipertensão arterial sistêmica), DM (diabetes mellitus), obesidade, cardiopatia, pneumopatia, hepatopatia, neoplasia, imunossupressão, se possível, devem ter exames laboratoriais básicos realizados: hemograma, eletrólitos, glicemia, GAMA-GT, PCR, transaminases, ureia e creatinina, bem como exames adicionais para seguimento: D-dímero, DHL, CPK e ferritina ${ }^{(29)}$.

Coleta de RT-PCR swab nasal e orofaringe: coletar de todos os suspeitos, conforme disponibilidade ${ }^{(29)}$.

Eletrocardiograma (ECG) ${ }^{(29)}$.

Raio-x de tórax e/ou tomografia computadorizada (TC) de tórax, conforme avaliação médica e condições do serviço de assistência ${ }^{(29)}$. 


\begin{tabular}{l} 
Terapia recomendada \\
\hline Hidroxicloroquina ${ }^{(18,19,27,29,30)}$ \\
$1^{\circ}$ dia: $400 \mathrm{mg}$, por via oral, de $12 / 12$ horas. \\
$2^{\circ}$ ao $7^{\circ}$ dia: $400 \mathrm{mg}$, por via oral, ao dia. \\
O tempo de uso pode variar segundo avaliação médica, podendo \\
durar de 5 até 10 dias, conforme o caso ${ }^{(18,19,27,29,30)}$. \\
Em caso de insuficiência renal: não necesita de ajuste se FG \\
$>30$ mg/dl. Se FG entre 15 e 30 mg/dl, não ministrar dose total. \\
Se $<15$ mg/dL, ministrar dose em dias alternados ${ }^{(29)}$. \\
Contraindicado em maculopatias e retinose pigmentar \\
Azitromicina ${ }^{(19,27,29)}$. \\
$1^{\circ}$ ao $5^{\circ}$ dia: 500 mg, por via oral, em dose única diária por 5 dias. \\
Gestantes, lactantes e crianças não têm contraindicação ao \\
uso do esquema recomendado! Crianças: 6.5 mg/Kg/dia de \\
hidroxicloroquina. Considerar a internação das grávidas de acordo \\
com a gravidade e a evolução do caso ${ }^{(29)}$. \\
Observações: caso os sintomas gripais predominem, na ausência \\
de manifestações específicas de COVID-19 (como náuseas, \\
diarreia e anosmia), associa-se oseltamivir 75 mg, por via oral, \\
de $12 / 12$ h por cinco dias ${ }^{(8)}$. \\
Contraindicação: reação alérgica ao uso de hidroxicloroquina e \\
azitromicina(29). \\
Atenção aos pacientes com cardiopatia, retinopatias, maculopatias, \\
insuficiência hepática, epilepsia ou hipersensibilidade à droga \\
\end{tabular}

Em pacientes hepatopatas ou não, o aumento das enzimas hepáticas pode ser pela ação viral, não sendo contraindicação absoluta para o esquema proposto, de preferência em regime de internação(29).

No caso de pacientes epiléticos, considerar internação e fazer o protocolo de acordo com decisão médica ${ }^{(31)}$.

\begin{tabular}{|c|c|}
\hline Terapia de acréscimo & Considerações \\
\hline $\begin{array}{l}\text { Tromboprofilaxia para todos os pacientes }{ }^{(19,33-36)} \text {. } \\
\text { Heparina não fracionada subcutânea: } 5 \text { mil Ul, de } 12 / 12 \mathrm{~h} \text {, se } \\
\text { peso }<70 \mathrm{~kg} \text {, e de } 8 / 8 \mathrm{~h} \text { se peso }>70 \mathrm{~kg} \text {, por } 7 \text { dias para todos } \\
\text { os pacientes e, por } 15 \text { dias, se apresentar alto risco de base } \\
\text { para trombose }{ }^{(19,33-36)} \text {. } \\
\text { Ou } \\
\text { Enoxaparina subcutânea, } 40 \mathrm{mg} \text {, se peso }<70 \mathrm{~kg} \text {, e } 60 \mathrm{mg} \text {, se } \\
\text { peso }>70 \mathrm{~kg} \text {, por } 7 \text { dias para todos os pacientes e, por } 15 \text { dias, } \\
\text { se apresentar alto risco de base para trombose }\end{array}$ & $\begin{array}{l}\text { Se insuficiência renal (FG <30, enoxaparina subcutânea } 20 \text { mg) } \\
(19,33-36) \text {. } \\
\text { Observações: caso D-dímero seja superior a } 1,5 \text { vezes o valor } \\
\text { de referência normal, iniciar imediatamente a anticoagulação, em } \\
\text { regime de internação, e fazer controle de D-dímero. } \\
\text { Nesses casos, se o acesso ao serviço de hospitalização não é } \\
\text { imediato, iniciar corticoide endovenoso ( } 80 \text { mg de metilprednisolona, } \\
\text { endovenosa). Se for possível, internar imediatamente o paciente. } \\
\text { Essa intervenção deve ser realizada pela equipe do hospital }{ }^{(29)} \text {. }\end{array}$ \\
\hline
\end{tabular}

\section{Considerações}

O esquema pode ser somente de três dias nos casos leves $^{(18,19,27,29,30)}$.

Evitar em insuficiência renal com $\mathrm{FG}<10 \mathrm{mg} / \mathrm{dL}^{(18,19,27,29,30)}$.

Não administrar com amiodarona, sotalol, pois aumenta os níveis de digoxina ${ }^{(29,30)}$.

$\mathrm{Na}$ ausência de hidroxicloroquina, pode-se optar pela cloroquina $^{(26,28,29,30)}$.

Pacientes acima de $60 \operatorname{anos}^{(29,32)}$

Pacientes com relato de cardiopatia e uso de medicações que podem prolongar o interno $\mathrm{QT}^{(29,32)}$.

Pacientes com frequência cardíaca inferior a 60 bpm $^{(32)}$.

Esse perfil de paciente deve, se possível, realizar ECG e, caso sejam encontradas alterações que possam predispor a arritmias, solicitar avaliação de médico cardiologista, de forma presencial ou por telemedicina. Após avaliação adequada, considerar a terapia com paciente hospitalizado. $\mathrm{Na}$ impossibilidade de acesso ao cardiologista ou telemedicina, calcular o intervalo QT corrigido pela frequência, de modo que ${ }^{(32)}$ :

Se intervalo QTC (QT corrigido) < $450 \mathrm{~ms}$, pode-se indicar hidroxicloroquina e azitromicina.

Se intervalo QTC for entre $450-500 \mathrm{~ms}$, fazer somente hidroxicloroquina.

Se intervalo QT > 500 ms, encaminhar para internação e não prescrever hidroxicloroquina e azitromicina.

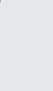




\section{Orientações pósprescrição}

Considerações

Pacientes estáveis, sem dispneia, alterações radiológicas e O início do tratamento não deve ser postergado, exceto se o comorbidades, podem ser encaminhados para o domicílio com médico julgar que o malefício seja maior que o benefício(19,33-36). esquema terapêutico prescrito(30).

Orientação de manutenção de isolamento social e domiciliar por

14 dias, usar quarto e banheiros próprios, separar objetos de uso pessoal do de outros moradores ${ }^{(8,30)}$.

Manter monitoramento e vigilância a distância via mensagens, ligações ou telemedicina( ${ }^{(8)}$.

Retornar ao serviço médico caso ocorram sinais de piora clínica( ${ }^{(8)}$.

Critérios para internação

Os critérios de internação seguirão os protocolos adotados pelas instituições hospitalares.

\section{DISCUSSÃO}

O Conselho Federal de Medicina do Brasil propôs, no dia 16 de abril de 2020, que o médico assistente deve considerar o uso de cloroquina e hidroxicloroquina em pacientes com sintomas leves no início do quadro clínico, nos casos em que tenham sido descartadas outras viroses e que tenham confirmado o diagnóstico de COVID19, em decisão compartilhada com o paciente.

O médico é recomendado a relatar ao doente que não existe, até o momento, nenhum trabalho que comprove o benefício do uso da droga, explicando os efeitos colaterais possíveis, e obter o consentimento livre e esclarecido do paciente ou dos familiares. Deve considerar, ainda, o uso em pacientes com sintomas importantes, mas ainda não com necessidade de cuidados intensivos, com ou sem necessidade de internação, e o uso compassivo em pacientes críticos recebendo cuidados intensivos ${ }^{(30)}$.

O entendimento sobre a COVID-19 ainda está incompleto, principalmente no que se refere a sequelas e resultados em longo prazo. Além disso, muito pouco foi escrito sobre as necessidades de reabilitação de pacientes com COVID-19 após a alta dos cuidados agudos. Já são relatadas na literatura sequelas cardíacas, neurológicas, respiratórias, cognitivas, psicológicas e em outros sistemas, como lesão renal, traqueal e outras. As variedades de sequelas vão desde aquelas associadas à doença viral até uma estadia prolongada na UTI. Além disso, muitos apresentam comorbidades pré-existentes, que podem potencializar tais sequelas ${ }^{(37)}$. Assim, diante dessas incertezas, o tratamento precoce parece ser importante para minimizar possíveis incapacidades e maximizar a função e qualidade de vida das pessoas afetadas ${ }^{(37)}$.

O manejo da COVID-19 determinado pelo Ministério da Saúde do Brasil foi modificado dia 20 de maio de 2020 e incluiu a utilização, em todo o território nacional, de hidroxicloroquinina/cloroquina e azitromicina na Fase I da COVID-19(29).

Vale destacar que a hidrocloroquina é um fármaco utilizado há mais de 70 anos, com dose e efeitos secundários bem descritos ${ }^{(26,30,38)}$. A Sociedade Brasileira de Reumatologia (SBR) alerta que a cloroquina e hidroxicloroquina, por serem medicamentos utilizados há muito tempo, possuem um perfil de segurança conhecido ${ }^{(30,38)}$. Os antimaláricos são considerados medicações imunomoduladoras e não imunossupressoras.

As reações colaterais mais comuns são relacionadas ao trato gastrointestinal, como desconforto abdominal, náuseas, vômitos e diarreia. Podem ocorrer, também, toxicidade ocular, cardíaca, neurológica e cutânea ${ }^{(30,38)}$. Além disso, paciente portadores de psoríase, porfiria e etilismo podem ser mais suscetíveis a eventos adversos cutâneos, geralmente sem gravidade. Em casos raros, pode ocorrer hemólise em pacientes com deficiência de glicose-6-fosfato-desidrogenase. A SBR ainda pede que seja dada atenção especial à interação com outras drogas, como macrolídeos, quinolonas, antivirais e antipsicóticos, o que pode levar a problemas cardíacos (alargamento do intervalo QT) $)^{(30,38)}$.

Os principais estudos que utilizaram hidroxicloroquina ou cloroquina na fase precoce apresentaram nos pacientes: melhor resposta clínica e radiológica, eliminação viral mais rápida, menor tempo de internação, redução da mortalidade, menos admissão na UTI e redução da probabilidade de sequelas ${ }^{(17-19,26,28)}$.

Outras alternativas terapêuticas estão sendo avaliadas em estudos prospectivos, baseados em medicamentos antivirais, anti-inflamatórios e imunomoduladores, terapia celular, antioxidantes e outras terapias. A terapia anticoagulante tem sido recomendada em pacientes com COVID-19 em estágio inicial ${ }^{(39)}$.

A infecção induzida por coronavírus SARS-CoV-2 pode estar associada a uma coagulopatia. A coagulopatia inicial da COVID-19 apresenta: elevação proeminente dos produtos de degradação do D-dímero e fibrina/fibrinogênio, 
enquanto anormalidades no tempo de protrombina, tempo parcial de tromboplastina e a contagem de plaquetas é relativamente incomum nas apresentações iniciais ${ }^{(3,34)}$. Sugere-se a triagem com testes de coagulação, incluindo a medição dos níveis de D-dímero e fibrinogênio ${ }^{(33,34)}$.

A coagulopatia associada ao COVID-19 deve ser gerenciada, como seria para qualquer paciente gravemente doente, seguindo a prática estabelecida de usar profilaxia tromboembólica para pacientes internados gravemente enfermos, e medidas de cuidados de suporte padrão para aqueles com coagulopatia ou coagulopatia intravascular disseminada induzida por sepse. No entanto há informações muito escassas sobre manejo do risco trombótico, distúrbios da coagulação e terapia anticoagulante em paciente com COVID-19(33,34).

Portanto, para a utilização em pacientes ambulatoriais devem ser discutidos os riscos e benefícios, sobretudo quando houver dúvidas sobre o diagnóstico com dengue, em que a utilização de anticoagulação pode ser catastrófica ${ }^{(30)}$.

Dois estudos randomizados brasileiros estão em andamento: um já iniciou (Coalisão Brasil 2) e está registrado no ClinicalTrials.gov ${ }^{(40)}$. É um estudo randomizado que está comparando uso de hidroxicloroquina versus hidroxicloroquina e azitromicina, com previsão de término do estudo em 30 de agosto de 2020, segundo o site Clinical Trials.

O outro estudo brasileiro é o RBR-3cbs3w, que irá recrutar pacientes ambulatoriais com doença leve, desenhado para incluir 1.300 pacientes, randomizando para hidroxicloroquina versus grupo de controle. Em 12 de abril de 2020 ainda não estava recrutando pacientes ${ }^{(41)}$.

Dessa forma, o presente estudo apresenta limitação importante, pois nenhum estudo randomizado com metodologia adequada, utilizando hidroxiclorqouina ou cloroquina na Fase 1 da COVID-19, foi publicado ainda.

Existem muitos ensaios clínicos em andamento na atualidade, mas provavelmente não serão divulgados os resultados, nem parciais, antes do segundo semestre de $2020^{(40,41)}$. Levando-se em consideração a gravidade da pandemia e os resultados iniciais dos estudos observacionais com letalidade de $0,7 \%$, como a do estudo francês utilizando hidroxicloroquina associada à azitromicina ${ }^{(19)}$, e também que a letalidade do Brasil está em $5,0 \%$, talvez muitas mortes poderiam ser evitadas adotando-se o protocolo proposto na atenção básica, além de reduzir o número de internação em enfermarias hospitalares e UTI(19).

\section{CONCLUSÃO}

Não existe evidência nível $1 \mathrm{~A}$ de que a associação de cloroquina ou hidroxicloroquina e azitromicina seja efetiva no tratamento da COVID-19.

Diante desses dados iniciais de estudos observacionais, que geram uma hipótese de que a hidroxicloroquina e azitromicina podem alterar o curso da doença, com provável diminuição da morbidade e, talvez, da mortalidade, certamente seria mais benéfico para os pacientes se lhes fosse oferecido esse tratamento off label, por se tratar de uma doença que tem uma alta letalidade e morbidade.

Principalmente por se tratar de duas drogas utilizadas há décadas, em vários cenários clínicos e com efeitos colaterais de pequena monta, com mortalidade raríssima.

Porém, a decisão de tomar ou não a medicação é do paciente, e o médico só deve prescrever após o paciente assinar o consentimento livre e esclarecido. A autonomia do médico e do paciente deve ser preservada, como sempre foi na Medicina. Ademais, estudos randomizados podem ser conduzidos para confirmar ou não essa hipótese.

\section{CONFLITOS DE INTERESSE}

Autores atestam que não há conflitos de interesses.

\section{CONTRIBUIÇÕES}

Sabas Carlos Vieira, Danilo Rafael da Silva Fontinele e Marina Bucar Barjud contribuíram com a elaboração e o delineamento do estudo; a aquisição, análise e interpretação dos dados; e a redação do manuscrito. Justino Moreira de Carvalho Junior, Lina Madeira Campos Melo, José Wilson Fonseca Filho, Gerson Luís Medina Prado e Alexandre Adad Alencar contribuíram com a aquisição, análise e interpretação dos dados; e a redação do manuscrito.

\section{REFERÊNCIAS}

1. Zhu N, Zhang D, Wang W, Li X, Yang B, Song J, et al. A novel coronavirus from patients with pneumonia in China, 2019. N Engl J Med. 2020;382(8):727-33. 
2. Holmes E. Novel 2019 coronavirus genome [Internet]. 2020 [Acesso em 2020 Maio 25]. Disponível em: http:// virological.org/t/novel-2019-coronavirus-genome/319

3. Jiang F, Deng L, Zhang L, Cai Y, Cheung CW, Xia Z. Review of the clinical characteristics of coronavirus disease 2019 (COVID-19). J Gen Intern Med. 2020;35(5):1545-49.

4. Lu R, Zhao X, Li J, Niu P, Yang B, Wu H, et al. Genomic characterisation and epidemiology of 2019 novel coronavirus: implications for virus origins and receptor binding. Lancet. 2020;395(10224):565-74.

5. WHO. Brote de enfermedad por coronavirus (COVID-19) [Internet]. 2020 [acesso em 2020 Maio 18]. Disponível em: https://www.who.int/es/emergencies/diseases/novel-coronavirus-2019

6. WHO. Coronavirus disease (COVID-19) pandemic [Internet]. 2020 [acesso em 2020 Jun 13]. Disponível em: https://www.who.int/emergencies/diseases/novel-coronavirus-2019

7. Ministério da Saúde (BR). Painel Coronavírus [Internet]. 2020 [acesso em 2020 Jun 13]. Disponível em: https://covid.saude.gov.br/

8. Lai CC, Shih TP, Ko WC, Tang HJ, Hsueh PR. Severe acute respiratory syndrome coronavirus 2 (SARSCoV-2) and coronavirus disease-2019 (COVID-19): the epidemic and the challenges. Int J Antimicrob Agents. 2020;55(3):105924.

9. Van Doremalen N, Bushmaker T, Morris DH, Holbrook MG, Gamble A, Williamson BN, et al. Aerosol and surface stability of SARS-CoV-2 as compared with SARS-CoV-1. N Engl J Med [Internet]. 2020 [acesso em 2020 Maio 18];382(16)1564-7. doi: 10.1056/NEJMc2004973

10. Ganyani T, Kremer C, Chen D, Torneri A, Faes C, Wallinga J, et al. Estimating the generation interval for COVID-19 based on symptom onset data. MedRxiv [Internet]. 2020 [acesso em 2020 Mar 17]. doi: 10.1101/2020.03.05.20031815

11. Wilson N, Kvalsvig A, Barnard LT, Baker MG. Case-fatality risk estimates for Covid-19 calculated by using a lag time for fatality. Emerg Infect Dis [Internet]. 2020 [acesso em 2020 Mar 17];26(6). doi: 10.3201/ eid2606.200320

12. Siddiqi HK, Mehra RM. COVID19 IIIness in native and immunosuppressed states: a clinical-therapeutic staging proposal. J Heart Lung Transplant. 2020;39(5):405-7.

13. Shaoqing L, Fang J, Wating S, Chang C, Jingli C, Wei M, et al. Clinical characteristics and outcomes of patients undergoing surgeries during the incubation period of COVID-19 infection. Clin Med. 2020;21:1-8.

14. Wynants L, Van Calster B, Collins GS, Riley RD, Heinze G, Schuit E, et al. Prediction models for diagnosis and prognosis of covid-19 infection: systematic review and critical appraisal. BMJ [Internet]. 2020 [acesso em 2020 Mar 17];369:m1328. doi:10.1136/bmj.m1328

15. Wang M, Cao R, Zhang L, Yang X, Liu J, Xu M, et al. Remdesivir and chloroquine effectively inhibit the recently emerged novel coronavirus (2019-nCoV) in vitro. Cell Res. 2020;30(3):269-71.

16. Cao B, Wang Y, Wen D, Liu W, Wang J, Fan G, et al. A trial of lopinavir-ritonavir in adults hospitalized with severe Covid-19. N Engl J Med. 2020;382(19):1787-99.

17. Gautret $P$, Lagier JC, Parola P, Hoang VT, Meddeb L, Mailhe M, et al. Hydroxychloroquine and azithromycin as a treatment of COVID-19: results of an open-label non-randomized clinical trial. Int J Antimicrob Agents. $2020 ; 105949$

18. Chen Z, Hu J, Zhang Z, Jiang S, Han S, Yan D, et al. Efficacy of hydroxychloroquine in patients with COVID-19: results of a randomized clinical trial. MedRxiv [Internet]. 2020 [acesso em 2020 Mar 17]. doi: 10.1101/2020.03.22.20040758

19. Million M, Lagier JC, Gautret P, Colson P, Fournier PE, Amrane S, et al. Early treatment of COVID-19 patients with hydroxychloroquine and azithromycin: a retrospective analysis of 1061 cases in Marseille, France. Travel Med Infect Dis [Internet]. 2020 [acesso em 2020 Mar 17];35:101738. doi: 10.1016/j.tmaid.2020.101738

20. Tang W, Cao Z, Han M, Wang Z, Chen J, Sun W, et al. Hydroxychloroquine in patients with mainly mild to moderate coronavirus disease 2019: open label, randomised controlled trial. BMJ. 2020;369:m1849. 
21. Borba MGS, Val FFA, Sampaio VS, Alexandre MAAA, Melo GC, Brito M, et al. Effect of high vs low doses of chloroquine diphosphate as adjunctive therapy for patients hospitalized with severe acute respiratory syndrome coronavirus 2 (SARS-CoV-2) infection: a randomized clinical trial. JAMA Netw Open [Internet]. 2020 [acesso em 2020 Mar 17];3(4):e208857. doi: 10.1001/jamanetworkopen.2020.8857

22. Geleris J, Sun Y, Platt J, Zucker J, Baldwin M, Hripcsak G, et al. Observational study of hydroxychloroquine in hospitalized patients with Covid-19. N Engl J Med. 2020 Maio 7; [Epub ahead of print].

23. Mehra MR, Desai SS, Ruschitzka F, Pate AN. Hydroxychloroquine or chloroquine with or without a macrolide for treatment of COVID-19: a multinational registry analysis. Lancet. 2020 Maio 22.

24. Chen J, Liu D, Liu L, Liu $P, X u Q$, Xia L, et al. A pilot study of hydroxychloroquine in treatment of patients with moderate COVID-19. Zhejiang Da Xue Xue Bao Yi Xue Ban. 2020;49(2):215-9.

25. Molina JM, Delaugerre C, Le Goff J, Mela-Lima B, Ponscarme D, Goldwirt L, et al. No evidence of rapid antiviral clearance or clinical benefit with the combination of hydroxychloroquine and azithromycin in patients with severe COVID-19 infection. Med Mal Infect. 2020;50(4):384.

26. Gao J, Tian Z, Yang X. Breakthrough: chloroquine phosphate has shown apparent efficacy in treatment of COVID-19 associated pneumonia in clinical studies. Biosci Trends. 2020;14(1):72-3.

27. Gautret P, Lagier JC, Parola P, Hoang VT, Meddeb L, Sevestre J, et al. Clinical and microbiological effect of a combination of hydroxychloroquine and azithromycin in 80 COVID-19 patients with at least a six-day follow up: a pilot observational study. Travel Med Infect Dis. 2020;34:101663.

28. Multicenter collaboration group of Department of Science and Technology of Guangdong Province and Health Commission of Guangdong Province for chloroquine in the treatment of novel coronavirus pneumonia. Expert consensus on chloroquine phosphate for the treatment of novel coronavirus pneumonia. Zhonghua Jie $\mathrm{He} \mathrm{He}$ Hu Xi Za Zhi. 2020;43(3):185-8.

29. Ministério da Saúde (BR). Orientações do ministério da saúde para tratamento medicamentoso precoce de pacientes com diagnóstico da Covid-19 [Internet]. 2020 [acesso em 2020 Maio 25]. Disponível em: https:// saude.gov.br/images/pdf/2020/June/18/COVID-FINAL-16JUNHO-LIvreto-1-V3.pdf

30. Conselho Federal de Medicina. Processo consulta CFM nº 8/2020 - Parecer CFM nº 4/2020. Tratamento de pacientes portadores de COVID-19 com cloroquina e hidroxicloroquina [Internet]. 2020 [acesso em $2020 \mathrm{Abr}$ 23]. Disponível em: https://sistemas.cfm.org.br/normas/visualizar/pareceres/BR/2020/4

31. Kuroda N. Epilepsy and COVID-19: associations and important considerations. Epilepsy Behav [Internet]. 2020 [acesso em 2020 Abr 23];108:107122. doi:10.1016/j.yebeh.2020.107122

32. Crediblemeds. Recommendations re: COVID-19 Treatments [Internet]. 2020 [acesso em 2020 Jun 13]. Disponível em: https://crediblemeds.org/blog/recommendations-re-covid-19-treatments/

33. Connors JM, Levy JH. COVID-19 and its implications for thrombosis and anticoagulation. Blood [Internet]. 2020 [acesso em 2020 Jun 13];135(23):2033-40. doi: 10.1182/blood.2020006000

34. Vivas D, Roldán V, Esteve-Pastor MA, Roldan I, Tello-Montoliu A, Ruiz-Nodar J, et al. Recomendaciones sobre el tratamiento antitrombótico durante la pandemia COVID-19. Posicionamiento del Grupo de Trabajo de Trombosis Cardiovascular de la Sociedad Española de Cardiología. Rev Esp Cardiol [Internet]. 2020 Abr 22 [acesso em 2020 Jun 13]. doi: 10.1016/j.recesp.2020.04.006

35. Li J, Li Y, Yang B, Wang H, Li L. Low-molecular-weight heparin treatment for acute lung injury / acute respiratory distress syndrome: a meta-analysis of randomized controlled trials. Int J Clin Exp Med. 2018;11(2)414-22.

36. Mousavi S, Moradi M, Khorshidahmad T, Motamedi M. Anti-inflammatory effects of heparin and its derivatives: a systematic review. Adv Pharmacol Sci. 2015;2015:507151.

37. Sheehy LM. Considerações para reabilitação pós-aguda para sobreviventes do COVID-19. JMIR Public Health Surveill [Internet]. 2020 [acesso em 2020 Jun 13];6(2):e19462. doi:10.2196/19462

38. Sociedade Brasileira de Reumatologia. COVID-19: SBR alerta sobre o uso de cloroquina e hidroxicloroquina [Internet]. 2020 [acesso 2020 Abr 23]. Disponível em: https://www.reumatologia.org.br/noticias/covid-19-sbralerta-sobre-o-uso-de-cloroquina-e-hidroxicloroquina/ 
39. Pascarella G, Strumia A, Piliego C, Bruno F, Del Buono R, Costa F, et al. COVID-19 diagnosis and management: a comprehensive. J Intern Med [Internet]. 2020 [acesso 2020 Abr 23];10.1111/joim.13091. doi: 10.1111/joim.13091

40. SEM; Hospital do Coracao; Hospital Sirio-Libanes; Brazilian Research In Intensive Care Network. Safety and Efficacy of Hydroxychloroquine Associated With Azithromycin in SARS-CoV2 Virus (Coalition Covid-19 Brasil II). Clinical Trials [Internet]. 2020 [acesso em 2020 Abr 19]. Disponivel em: https://clinicaltrials.gov/ct2/show/ NCT04321278?term=HYDROXYCHLOROQUINE\&cntry=BR\&draw=2

41. Ministério da Saúde (BR). RBR-3cbs3w: estudo clínico randomizado, pragmático, duplo-cego, controlado por placebo, avaliando Hidroxicloroquina para prevenção de hospitalização e complicações respiratórias em pacientes ambulatoriais com diagnóstico confirmado ou provável de COVID-19. Registro Brasileiro Ensaios Clín [Internet]. 2020 Abr 2 [acesso em 2020 Abr 19]. Disponível em: http://www.ensaiosclinicos.gov.br/rg/RBR$3 \mathrm{cbs} 3 \mathrm{w} /$

\section{Endereço do primeiro autor:}

Sabas Carlos Vieira

Universidade Federal do Piauí - Centro de Ciências da Saúde

Rua Félix Pacheco, 2159/ sala 305

Bairro: Centro

CEP.: 64001-160 - Teresina - PI - Brasil

E-mail: drsabasvieira@gmail.com

\section{Endereço para correspondência:}

Danilo Rafael da Silva Fontinele

Rua Olavo Bilac, 2335

Bairro: Centro

CEP: 64015-017 - Teresina - PI - Brasil

E-mail: drsilvafontinele@gmail.com

Como citar: Vieira SC, Fontinele DRS, Barjud MB, Carvalho JM Junior, Melo LMC, Fonseca JW Filho, et al. Tratamento precoce para COVID-19 baseado em evidência científica. Rev Bras Promoç Saúde. 2020;33:10993. 